\title{
Connections between Latin Squares and Geometries
}

\author{
Md. Arshaduzzaman
}

\begin{abstract}
The present paper deals with Latin squares, orthogonal Latin squares, mutually Orthogonal Latin squares, close connections between Latin squares and finite geometries. Moreover the great mathematician Leonhard Euler introduced Latin squares in 1783 as a "nouveau espece de carres magiques", a new kind of magic squares. He also defined what he meant by orthogonal Latin squares, which led to a famous conjecture of his that went unsolved for over 100 years. In 1900, G. Tarry proved a particular case of the conjecture. It was shown in 1960 by Bose, Shrikhande, and Parker that, except for this one case, the conjecture was false. Key words and phrases: Latin squares, Smoking machine, Statistical experiments, group table, orthogona Latin square.
\end{abstract}

\section{Introduction}

The systematic development of Latin squares started with Euler (1779) and was carried on by Cayley (1877-1890) who showed that the multiplication table of a group is an Appropriately bordered special Latin square. In the 1930's the concept arose once again in the guise of multiplication tables when the theory of quasigroups and loops began to be developed as a generalization of the group concept. Latin squares played an important role in the foundations of finite geometries, a subject which was also in development at this time. Also in the 1930's, a big application area for Latin squares was opened by R.A.Fisher who used them and other combinatorial structures in the design of statistical experiments. Latin squares first arose with parlor games such as the problem of arranging the jacks, queens, kings, and aces of a pack of cards in a $4 \times 4$ array so that each row and each column contains one card from each suit and one card from each rank. In 1779, Leonard Euler posed the following famous problem of the 36 officers from six ranks and six regiments. He claimed it was impossible to arrange these officers on parade in a $6 \times 6$ square so that each row and each column contains one officer from each rank and one from each regiment.

Recently, statisticians have found Latin squares useful in designing experiments, and mathematicians have found close connections between Latin squares and finite geometries.

\section{Some Definitions and Auxiliary Results}

This section contains some definitions and auxiliary results.

Definition 2.1. ( Latin Squares ) Let $S$ be a set with $n$ elements. Then a Latin square $L=\left(l_{i j}\right)$, of order $\mathrm{n}$ based on $S$, is an $n \times n$ array of the elements of $\mathrm{S}$ such that each element appears, exactly once in each row and once in each column.

For example, Table 2.1. illustrates a Latin square of order 3 based on $\{a, b, c\}$.

Table 2.1. A Latin square

\begin{tabular}{|lll}
$a$ & $b$ & $c$ \\
$c$ & $a$ & $b$ \\
$b$ & $c$ & $a$ \\
\hline
\end{tabular}

Theorem 2.2. The table for any finite group $(G,+)$ of order $n$ is a Latin square of order $n$ based on $G$.

Proof. We write the operation in $G$ as addition, even though the result holds if $G$ is not commutative.

Suppose that two elements in one row are equal. Then $x_{i}+x_{J}=x_{i}+x_{k}$ for some $x_{i}, x_{i}, x_{k} \in G$. Since $G$ is a group, $x_{i}$ has an inverse $\left(-x_{i}\right)$ such that $\left(-x_{i}\right)+x_{i}=0$. Hence $\left(-x_{\mathrm{i}}\right)+\left(x_{i}+x_{j}\right)=\left(-x_{i}\right)+\left(x_{i}+x_{k}\right)$, and, since the operation is associative, we have $x_{j}=x_{k}$. Therefore, an element cannot appear twice in the same row. Similarly, an element cannot appear twice in some column, and the table is a Latin square.

Given any Latin square, we can permute the rows among themselves also the columns among themselves and we still have a Latin square. For example, the addition table for $\mathbf{Z}_{2} \times \mathbf{Z}_{2}$ is a Latin square of order 4 . If we interchange the first and third columns and replace $(0,0)$ by a, $(0,1)$ by b, $(1,0)$ by $c$, and $(1,1)$ by $\mathrm{d}$, we obtain another Latin square of order 4 based on $\{a, b, \mathrm{c}, d\}$. These are illustrated in Table 2.2.

Table 2.2. Latin squares of order four 


$\left.\begin{array}{|llll}(0,0) & (0,1) & (1,0) & (1,1) \\
(0,1) & (0,0) & (1,1) & (1,0) \\
(1,0) & (1,1) & (0,0) & (0,1) \\
(1,1) & (1,0) & (0,1) & (0,0)\end{array}\right] \quad$\begin{tabular}{llll|}
$c$ & $b$ & $a$ & $d$ \\
$d$ & $a$ & $b$ & $c$ \\
$a$ & $d$ & $c$ & $b$ \\
$b$ & $c$ & $d$ & $a$ \\
\hline
\end{tabular}

Useful in designing statistical experiments because they can show how an experiment can be arranged so as to reduce the errors without making the experiment too large or too complicated. See Mann [1] for more complete details.

Suppose you wanted to compare the yields of three varieties of hybrid corn. You have a rectangular test plot, but you are not sure that the fertility of the soil is the same everywhere. You could divide up the land into nine rectangular regions and plant the three varieties, $a, b$, and $c$, in the form of the Latin square in Table 1.1. Then if one row were more fertile than the others, the Latin square would reduce the error that this might cause. In fact, if the soil fertility was a linear function of the coordinates of the plot, the Latin square arrangement would minimize the error.

Of course the error could be reduced by subdividing the plot into a large number of pieces and planting the varieties at random. But this would make it much more difficult to sow and harvest.

Example 2.3. A smoking machine is used to test the tar content of four brands of cigarettes; the machine has four ports so that four cigarettes can be smoked simultaneously. However, these four ports might not be identical and that might affect the measurements of the tar content. Also, if four runs were made on the machine, testing one brand at a time, the humidity could change, thus affecting the results.

Show how to reduce the errors due to the different ports and the different runs by using a Latin square to design the experiment.

Solution. If $a, b, c, d$ are the four brands, we can use one of the Latin squares of order 4 that we have constructed. Table 2.3. illustrates which brand should be tested at each port during each of the four runs.

Table 2.3. The design of the smoking experiment

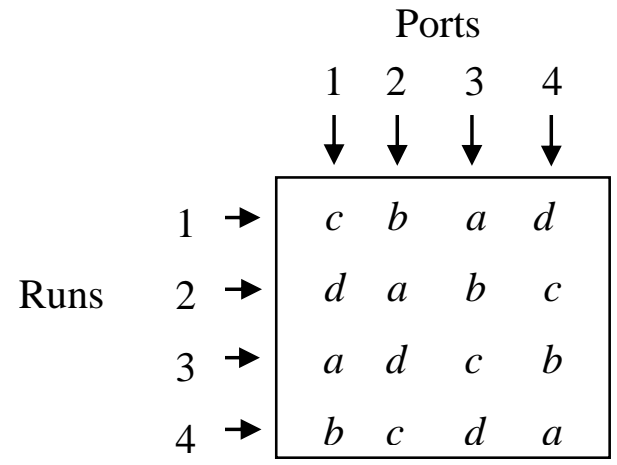

Not all Latin squares can be obtained from a group table, even if we allow permutations of the rows and columns.

Example 2.4. Show that the Latin square illustrated in Table 2.4. cannot be obtained from a group table.

Table 2.4.

\begin{tabular}{|cc:ccc|}
\hline$A$ & $B$ & $C$ & $D$ & $E$ \\
$B$ & $A$ & $E$ & $C$ & $D$ \\
\hdashline$C$ & $D$ & $A$ & $E$ & $B$ \\
$D$ & $E$ & $B$ & $A$ & $C$ \\
$E$ & $C$ & $D$ & $B$ & $A$ \\
\hline
\end{tabular}

Solution. We know that all groups of order 5 are cyclic and are isomorphic to $\left(\mathbf{Z}_{5},+\right)$. Suppose that the Latin 
square in Table 2.4. could be obtained from the addition table of $\mathbf{Z}_{5}$. Since permutations are reversible, it follows that the rows and columns of this square could be permuted to obtain the table of $\mathbf{Z}_{5}$. The four elements in the left hand top corner would be taken into four elements forming a rectangle in $\mathbf{Z}_{5}$, as shown in table 2.5 . Then we would have $p+r=A, q+r=B, p+s=B$, and $q+s=A$ for some $p, q, r, s \in \mathbf{Z}_{5}$, where $p \neq q$ and $r \neq s$. Hence $p+r=A=q+s$ and $r=B=p+s$. Adding, we have $p+q+2 r=p+q+2 s$ and $2 r=2 s$. before, $6 r=6 s$, which implies $r=s$ in $\mathbf{Z}_{5}$, which is a contradiction.

Table 2.5.

\begin{tabular}{c|cc}
+ &..$p$ & $. . q .$. \\
\hline & & \\
. & $A$ & $B$ \\
. & & $A$ \\
$r$ & $B$ & \\
. & &
\end{tabular}

\section{Orthogonal Latin Squares}

Suppose that, in our corn field, besides testing the yields of three varieties of corn, we also wanted to test the effects of three fertilizers on the corn. We could do this in the same experiment by arranging the fertilizers on the nine plots so that each of the three fertilizers was used once on each variety of corn and so that the different fertilizers themselves were arranged in a Latin square of order 3.

Let $a, b, c$ be three varieties of corn and $A, B, C$ be three types of fertilizer. Then the two Latin squares in Table 2.6. could be superimposed to form the design in Table 2.7. In this table, each variety of corn and each type of fertilizer appears exactly once in each row and in each column. Furthermore, each type of fertilizer is used exactly once with each variety of corn. This table could be used to design the experiment. For example, in the top left section of our test plot, we would plant variety " $a$ " and use fertilizer " $A$ ".

Table 2.6.

\begin{tabular}{|lll}
$a$ & $b$ & $c$ \\
$c$ & $a$ & $b$ \\
$b$ & $c$ & $a$ \\
$B$ & $A$ & $B$ \\
\hline
\end{tabular}

Table 2.7.

\begin{tabular}{|ccc|}
$a A$ & $b B$ & $c C$ \\
$c B$ & $a C$ & $b A$ \\
$b C$ & $c A$ & $a B$ \\
\hline
\end{tabular}

Definition 2.5. Two Latin squares of order $\mathrm{n}$ are called orthogonal if, when the squares are superimposed, each element of the first square occurs exactly once with each element of the second square.

The two Latin squares in Table 2.6. are orthogonal.

Although it is easy to construct Latin squares of any order, the construction of orthogonal Latin squares can be a difficult problem. At this point the reader should try to construct two orthogonal Latin squares of order 4 .

Going back to our field of corn and fertilizers, could we use the same trick again to test the effect of three insecticides by choosing another Latin square of order 3 orthogonal to the first two? It can be proved that it is impossible to find such a Latin square. However, if we have four types of corn, fertilizer, and insecticide, we show, using Theorem 2.8, how they could be distributed on a $4 \times 4$ plot using three Latin squares of order 4 orthogonal to each other.

Definition 2.6. If $L_{1}, \ldots, L_{r}$ are Latin squares of order $\mathrm{n}$ such that $L_{i}$ is orthogonal to $L_{j}$ for all $i \neq j$, then $\left(L_{1}, \ldots\right.$, $L_{r}$ ) is called a set of $r$ mutually orthogonal Latin squares of order $n$.

We show how to construct $n-1$ mutually orthogonal Latin squares of order $n$ from a finite field with $n$ elements. We know that a finite field has a prime power number of elements, and we are able to construct such squares for $n=2,3,4,5,7,8,9,11,13,16,17, \ldots$ etc.

Let $\mathbf{G F}(n)=\left\{x_{0}, x_{1}, x_{2}, \ldots, x_{n-1}\right\}$ be a finite field of order $n-p^{m}$ where $x_{0}=0$ and $x_{1}=1$. Let $L_{1}=\left(a_{i j}^{1}\right)$ be the Latin square of order $\mathrm{n}$ that is the addition table of $\mathbf{G F}(n)$. Then

$$
a_{i j}^{1}=x_{i}+x_{j} \text { for } 0 \leq i \leq \mathrm{n}-1,0 \leq j \leq n-1 .
$$

Proposition 2.7. Define the squares $L_{k}=\left(a_{i j}^{1}\right)$, for $1 \leq k \leq n-1$, 
By $\quad a_{i j}^{1}=x_{k} \cdot x_{i}+x_{j}$ for $0 \leq i \leq n-1,0 \leq j \leq n-1$.

Then $L_{k}$ is a Latin square of order $n$ for $1 \leq k \leq n-1$ based on $\mathbf{G F}(n)$.

Proof. The difference between two elements in the $i$ th row is

$$
\begin{aligned}
& a_{i j}^{k}=a_{i q}^{k}=\left(x_{k} \cdot x_{i}+x_{j}\right)-\left(x_{k} \cdot x_{i}+x_{q}\right) \\
& =x_{j}-x_{q} \neq 0, \quad \text { if } j \neq q .
\end{aligned}
$$

Hence each row is a permutation of $\mathbf{G F}(n)$.

The difference between two elements in the $j$ th column is

$$
\begin{aligned}
& a_{i j}^{k}=a_{r j}^{k}=\left(x_{k} \cdot x_{i}+x_{j}\right)-\left(x_{k} \cdot x_{r}+x_{j}\right) \\
& =x_{k} \cdot\left(x_{i}-x_{r}\right) \neq 0, \quad \text { if } i \neq r, \quad \text { since } x_{k} \neq 0 \quad \text { and } x_{i} \neq x_{r} .
\end{aligned}
$$

Hence each column is a permutation of $\mathbf{G F}(n)$ and $L_{k}$ is a Latin square of order $n$.

Theorem 2.8. $\left\{L_{1}, L_{2}, \ldots, L_{n-1}\right\}$ is a mutually orthogonal set of tin squares of order $n=p^{m}$.

Proof. We have to prove that $L_{k}$ is orthogonal to $L_{1}$ for all $k \neq 1$.

Suppose that when $L_{k}$ is superimposed on $L_{1}$, the pair of elements in the $(i, j)$ th position is the same as the pair in the $(r, q)$ th position. That is, $\left(a_{i j}^{k}, a_{i j}^{l}\right)=\left(a_{r q}^{k}, a_{r q}^{l}\right)$ or $a_{i j}^{k}=a_{r q}^{k}$ and $a_{i j}^{l}=a_{r q}^{l}$. Hence $x_{k} \cdot x_{i}+x_{j}=x_{k} \cdot x_{r}+x_{q}$ and $x_{l} \cdot x_{i}+x_{j}=x_{l} \cdot x_{r}+x_{q}$. Subtracting, we have $\left(x_{k}-x_{l}\right) \cdot x_{i}=\left(x_{k}-x_{l}\right) \cdot x_{r}$ or $\left(x_{k}-\right.$ $\left.x_{l}\right) \cdot\left(x_{i}-x_{r}\right)=0$. Now the field $\mathbf{G F}(\mathrm{n})$ has no zero divisors; thus either $x_{k}=x_{1}$ or $x_{1}=x_{r}$. Hence either $k=l$ or $i=r$. But $k \neq l$ and we know from position 1.7 that two elements in the same row of $L_{k}$ or $L_{l}$ cannot be equal; therefore, $i \neq r$.

This contradiction proves that when $L_{k}$ and $L_{l}$ are superimposed, all the pairs of elements occurring are different. Each element of the first square appears $\mathrm{n}$ times and hence must occur with all the $\mathrm{n}$ different elements of the second square. Therefore, $L_{k}$ is orthogonal to $L_{l}$, if $k \neq l$.

If we start with $\mathbf{Z}_{3}$ and perform the above construction we obtain the 3 mutually orthogonal Latin squares of order 3 given in Table 2.8 .

Table 2.8. Two orthogonal Latin squares

\begin{tabular}{|lll|}
\hline 0 & 1 & 2 \\
1 & 2 & 0 \\
2 & 0 & 1
\end{tabular}$\quad\left[\begin{array}{lll|}0 & 1 & 2 \\
2 & 0 & 1 \\
1 & 2 & 0 \\
\hline\end{array}\right.$

Example 2.9. A drug company wishes to produce a new cold remedy by combining a decongestant, an antihistamine, and ${ }^{1}$ a pain reliever. It plans to test variơ⿱中⿰㇀丶㇀ antihistamines, and three pain relievers on four groups of subjects each day from Monday to Thursday. Furthermore, each type of ingredient should also be compared with a placebo. Design this test so as to reduce the effects due to differences between the subject groups and the different days.

Solution. We can use the three mutually orthogonal Latin squares instructed in the previous example to design this experiment.

Make up the drugs given to each group using Table 2.9. The letter in the first position refers to the

\begin{tabular}{|c|c|c|c|c|c|}
\hline & & Mon & Tue & Wed & Thu \\
\hline & A & $a a a$ & $b b b$ & $c c c$ & $d d d$ \\
\hline Subject & $B$ & $b c d$ & $a d c$ & $d a b$ & $c b a$ \\
\hline \multirow[t]{2}{*}{ Groups } & $C$ & $c d b$ & $d c a$ & $a b d$ & $b a c$ \\
\hline & $D$ & $d b c$ & cad & $b d a$ & $a c b$ \\
\hline
\end{tabular}
decongestant, the second to the antihistamine, and the third to the pain reliever. The letter " $a$ " refers to a placebo, and " $b$ ", " $c$ " and " $d$ " refer to the three different types of ingredients.

Table 2.9. Testing three different drugs

We recognize Euler's problem of the 36 officers on parade, as the problem of constructing two orthogonal Latin squares of order 6. Euler not only conjectured that this problem as impossible to solve, but he also conjectured that it was impossible to find two orthogonal Latin squares of order $n$, whenever $n \equiv 2 \bmod 4$.

No prime power is congruent to 2 modulo 4; therefore, we cannot use the method of Theorem 2.8. to 
construct any of these squares. In 1899, G. Tarry, by exhaustive enumeration, proved that the problem of the 36 officers was insoluble. However, in 1959, Euler's general conjecture was shown to be false, and, in fact, Bose, Shrikhande and Parker proved that there exist at least two orthogonal Latin squares of order $n$, for any $n>$ 6. Hence Proposition 2.7 is by no means the only way of constructing orthogonal Latin squares. Denes and Keedwell [2] give a comprehensive survey of all the known results on Latin squares up to the time of its publication.

\section{Finite Geometries}

\section{Main Results Now we prove our main results.}

This method of constructing $n-1$ mutually orthogonal Latin squares of order $\mathrm{n}$ over $\mathbf{G F}(n)$ was originally given by R. C. Bose [3]. In the same paper, Bose showed that there is a very close connection between orthogonal Latin squares and geometries with a finite number of points and lines.

The geometries that we consider are called affine planes.

Definition 2.10. An affine plane consists of a set, $P$, of points, together with a set, $L$, of subsets of $P$ called lines. The points and lines must satisfy the following incidence axioms.

(i) Any two distinct points lie on exactly one line,

(ii) For each line $l$ and point $x$ not on $l$, there exists a unique line $\mathrm{m}$ containing $\mathrm{x}$ and not meeting $l$.

(iii) There exist three points not lying on a line.

We can define an equivalence relation of parallelism, //, on the set of lines $L$, by defining $l / / m$ if $l=m$ or $l$ and $\mathrm{m}$ contain no common point. Axiom (ii) then states that through each point there is a unique line parallel to any other line.

The points and lines in the Euclidean plane $\mathbf{R}^{2}$ form such a geometry with an

infinite number of points.

If the geometry has only a finite number of points, it can be shown that there exists an integer $n$ such that the geometry contains $n^{2}$ points and $n^{2}+n$ lines, and that each line contains $n$ points, while each point lies on $n+1$ lines. Such a finite geometry is called an affine plane of order $n$. In an affine plane of order $n$ there are $n+1$ parallelism classes.

The diagram in Figure 2.1 shows an affine plane of order 2 in which $P=\{a, b, c, d)$ and $L=\{\{a, b\},\{c, d),\{a$, $c),\{b, c\},\{b, d\},\{a, d\}\}$.

Bose showed that an affine plane of order $\mathrm{n}$ produces a complete set of $n-1$ mutually orthogonal Latin squares of order $n$, and conversely, that each set of $n-1$ mutually orthogonal Latin squares of order $n$ defines an affine plane of order $n$.

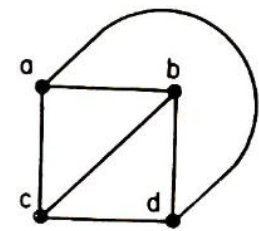

Figure 2.10. An affine plane with four points.

\section{Conclusion:}

Hence an affine plane of order $\mathrm{n}$, it is possible to construct a projective plane of order $\mathrm{n}$ by adding a "line at infinity" containing $\mathrm{n}+1$ points corresponding to each parallelism class, so that parallel lines intersect on the line at infinity. The projective plane of order $n$ has $n^{2}+n+1$ points and $\quad n^{2}+n+1$ lines. Furthermore, any projective plane give rise to an affine plane by taking one line to be the line at infinity. Hence the existence of a projective plane of order $\mathrm{n}$ is equivalent to the existence of an affine plane of the same order.

\section{References}

[1] Mann, H. B., Analysis and Design of Experiments, Dover, New York, 1949

[2] Denes, J. and A. D. Keedwell, Latin Squares and their Applications, Academic $\quad$ Press, New York, 1974.

[3] Bose, Ray Chandra, "On the Application of the Properties of Galois Fields to the Problem of Construction of Hyper-Graeco-Latin Squares," Sankhya (The Indian Journal of Statistics), 3, 323-338(1938).

[4] Ball, W. W. Rouse, Mathematical Recreations and Essays, rev. ed. Macmillan, London, 1939.

[5] Horadam, A. F., A guide to Undergraduate Projective Geometry, Pergamon Press Australia, Rushcutters Bay, N.S.W., 1970.

[6] Bailey, R.A. (2008). "6 Row-Column designs and 9 More about Latin squares". Design of Comparative Experiments. Cambridge University Press. ISBN 978-0-521-68357-9.

[7] Dénes, J. H.; Keedwell, A. D. (1991). Latin squares: New developments in the theory and applications. Annals of Discrete Mathematics 46. Amsterdam: Academic Press. ISBN 0-444-88899-3.

[8] Hinkelmann, Klaus; Kempthorne, Oscar (2008). Design and Analysis of Experiments. I, II （Second ed.). Wiley. ISBN 9780-470-38551-7.

[9] Laywine, Charles F.; Mullen, Gary L. (1998). Discrete mathematics using Latin squares. Wiley- Interscience Series in Discrete Mathematics and Optimization. New York: John Wiley \& Sons, ISBN 0-471-24064-8. 
[10] Cayley, A. "On Latin Squares." Oxford Cambridge Dublin Messenger Math. 19, 135-137, 1890.

[11] McKay, B. D. and Wanless, I. M. "On the Number of Latin Squares." Ann. Combin. 9, 335-344, 2005.

[12] R. C. Bose, S. S. Shrikhande, E. T. Parker, Further Results on the Construction of Mutually Orthogonal Latin Squares and the Falsity of Euler's Conjecture, Canadian Journal of Mathematics, vol. 12 (1960), pp. 189-203.

[13] C. F. Laywine and C.M. Mullen, Discrete Mathematics Using Latin Squares, Wiley Interscience, 1998. 